\title{
Panorama profesional en México, un escenario limitado para el estudiantado universitario
}

\section{Professional prospect in Mexico, a limited stage for college students}

\author{
Omar Iván Gavotto-Nogales ${ }^{1}$ \\ Centro Regional de Formación Docente e Investigación \\ Educativa del Estado de Sonora \\ Hermosillo, Sonora, México \\ ogavotto@crfdies.edu.mx \\ Lidia Isabel Castellanos-Pierra ${ }^{2}$ \\ Centro Regional de Formación Docente e Investigación \\ Educativa del Estado de Sonora \\ Hermosillo, Sonora, México \\ lidiaisabelcp@yahoo.es
}

\section{Recibido: 12 febrero 2014 Aceptado: 4 abril 2015 Corregido: 11 mayo 2015}

\begin{abstract}
Resumen: El objetivo del estudio es contrastar el panorama laboral en México y la factibilidad para obtener un empleo en el campo profesional, al finalizar los estudios universitarios. Desde una perspectiva crítica se señala que el costo-beneficio hace imposible sustentar las políticas y estrategias que han sido objeto de la educación en México; sin embargo, se promueve la participación del sector privado en este ámbito, lo que puede representar un factor que facilita el ingreso a la universidad, pero también puede limitar el acceso a este nivel educativo. Resultando la teoría de Thurow más apropiada para explicar la problemática del desempleo que existe y una sobrepoblación de profesionales, puesto que no es suficiente la inversión en el capital humano para obtener un empleo, si no se cuenta con la oferta laboral pertinente y suficiente, se concluye que el panorama profesional en México representa un escenario limitado para la población estudiantil universitaria, con base en los siguientes argumentos: a) Un 75\% del desempleo en México corresponde a personas con estudios universitarios; b) La mayoría de las personas ocupadas o empleadas realizan actividades que no corresponden a su profesión; c) La inversión en el capital humano no representa para gran cantidad de profesionistas un proyecto de recuperación económica a corto plazo; y d) La población de jóvenes en edad de estudiar la universidad se incrementa cada año; no obstante, las universidades públicas no tienen la capacidad para facilitar el ingreso a la totalidad de sus aspirantes.
\end{abstract}

Palabras clave: Empleo, oportunidades, estudiantes.

1 Es egresado de la Licenciatura en Educación Física en la Escuela Normal de Educación Física en el estado de Sonora en México. Obtuvo el grado de Maestría en Educación en la Universidad de Montemorelos y la Maestría en Innovación para la Calidad de la Educación Física en la Escuela Normal Estatal de Especialización, el Doctorado en Ciencias de la Educación en la Universidad Iberoamericana, y el Doctorado en Cultura Física y Deporte en el CIES-UNEPROP, titulándose de ambos doctorados con mención honorífica. Es un pedagogo especialista en la educación basada en competencias y diseño curricular, autor de varios libros: Crítica a la Educación Práctica y a la Práctica de la Educación en México; La Evaluación de Competencias Educativas; y Transformando la Educación Desde la Práctica Docente. Se ha desempeñado como maestro de nivel superior en 22 universidades del noroeste de México. Actualmente imparte conferencias a nivel internacional y trabaja de tiempo completo con el Centro Regional de Formación Docente e Investigación Educativa del Estado de Sonora.

2 Graduada en el año 2004 con título de oro y con el promedio más alto de su generación en la Licenciatura en Historia del Arte de la Universidad de Oriente en Santiago de Cuba, Cuba. Obtuvo en dicha institución el título de Máster en Ciencias de la Educación Superior. Se ha desempeñado como docente del Claustro de Historia del Arte de la Universidad de Oriente, como profesora de asignatura del Departamento de Historia y Antropología de la Universidad de Sonora y como profesora de la Licenciatura en Educación de la Universidad del Valle de México, Campus Hermosillo. Actualmente es candidata a doctora del Doctorado en Educación en el Centro Universitario Hermosillo, en México. 


\begin{abstract}
The aim of the study is to compare the employment landscape in Mexico and the feasibility to get a job in the professional field, after university studies. From a critical perspective it is stated that the cost-benefit makes it impossible to sustain policies and strategies that have been the subject of education in Mexico, however, the private sector in this area is promoted, which can be a factor that facilitates college entrance but can also limit access to this level. Thurow's theory is the most appropriate one to explain the problem of unemployment that exists and an overpopulation of professionals, since the investment in human capital is not enough to get a job, if you do not have the relevant and sufficient labor supply. It is concluded that the professional scene in Mexico, represents a limited scenario for college students, based on the following arguments: a) 75\% of unemployed in Mexico is represented by people with university degrees; b) Most busy professionals or employees are engaged in activities that do not correspond to their profession; c) Investment in human capital does not represent an economic recovery in the short-term for many professionals; d) The population of school-age young college increases each year, however, public universities are unable to facilitate entry to all applicants.
\end{abstract}

Keywords: Employment, opportunities, students.

En el artículo se analiza la relación oferta y demanda laboral profesional con base en datos demográficos, en las principales áreas de ocupación, cobertura educativa y tendencias de las preferencias del estudiantado. Se cuestiona la inversión en capital humano como la principal variable para obtener un empleo bien remunerado en el campo profesional.

El objetivo del estudio es contrastar el panorama laboral en México y la factibilidad para obtener un empleo en el campo profesional al finalizar los estudios universitarios. Desde una perspectiva crítica, se señala que el costo-beneficio hace imposible sustentar las políticas y estrategias que han sido objeto de la educación universitaria en México; sin embargo, se promueve la participación del sector privado en este ámbito, lo que puede representar un factor que facilita el ingreso a la universidad, pero que también puede limitar el acceso a este nivel educativo.

Por medio de una investigación documental de informes presentados por órganos gubernamentales y no gubernamentales, se revisaron diversas fuentes que ofrecen datos empíricos confiables para el objetivo del estudio. Por tal razón, el estudio es descriptivo, ex post facto y longitudinal.

En los últimos años, tanto en México como en Latinoamérica se han presentado profundas transformaciones en el contexto de las instituciones de educación superior. La universidad en México se proyecta como una institución que proporciona formación a una gran mayoría de la población a lo largo de toda la vida (PROFLEX, 2010). A medida que México avanza hacia el desarrollo de la nueva sociedad del conocimiento, resulta cada vez más importante disponer de una educación superior de mayor calidad, que favorezca la creación y distribución de los conocimientos y tecnologías que demanda una sociedad globalizada. Sin embargo, México, al ser un país con una extensión territorial de $1,964,375 \mathbf{~ k m}^{2}$ presenta grandes contrastes y diferentes oportunidades para sus habitantes. En la última década, México ha sido señalado como un país que no responde a las expectativas educativas debido al bajo rendimiento académico de sus estudiantes y población graduada en todos los niveles educativos.

De acuerdo con el Consejo Nacional de Población, México tiene 118,395,054 habitantes, de los cuales las mujeres representan el $51 \%$ con $60,584,099$ y los hombres un $48.8 \%$, con 
57,810,955. Actualmente, se tiene una esperanza de vida de 74.5 años a nivel nacional. Desde una perspectiva de género, la esperanza de vida para mujeres es de 77 años y para los hombres de 71 años (CONAPO, 2014). Se proyecta para el 2050 un incremento notable de la población mexicana a 150,837,517,32 millones más, y la esperanza de vida a nivel nacional será aproximadamente de 80 años. Se estima que al incrementarse la esperanza de vida, la población mexicana tendrá una mayor oportunidad para contribuir con el desarrollo social y económico del país. También, se estima que el crecimiento continuará con estas tendencias en los próximos 60 años, debido a la tasa de natalidad, la movilidad laboral y la inversión económica nacional y extranjera.

De acuerdo con la Secretaría de Educación Superior de la Secretaría de Educación Pública, la escasez de recursos es un rasgo característico de las instituciones de educación superior en México y en el ámbito latinoamericano. Las causas de su permanente austeridad se deben, en mucho, a la carencia de recursos fiscales, lo que, a su vez, responde a la competencia por el financiamiento de diversas necesidades sectoriales, tales como la salud, el orden público y el combate a la pobreza, entre otras (Tuirán y Muñoz, 2009). En ese contexto, el sistema educativo nacional ha emprendido transformaciones profundas en sus sistemas de financiamiento para la educación superior y para ampliar su cobertura.

De acuerdo con Tuirán y Muñoz (2009) en el documento La educación superior: Retos, desafios y prioridades para el futuro, donde se describen las principales debilidades que tiene la educación superior en México en la actualidad, destaca la insuficiente cobertura ante una demanda creciente; la inequidad en el acceso en perjuicio de personas jóvenes de estratos socioeconómicos bajos; los circuitos diferenciados de calidad y la débil pertinencia y limitada vinculación de las instituciones de educación superior con el sector productivo.

Aunque la cobertura en educación superior asciende paulatinamente, se encuentra, actualmente, una cobertura aproximada de un $30.9 \%$ de la población en edad de cursar la universidad; no obstante, a pesar de este incremento, de la matrícula sigue siendo baja (Tuirán y Muñoz, 2009).

En los últimos ciclos escolares algunas instituciones públicas de educación superior han establecido políticas de "numerus clausus" en la admisión de estudiantes, determinando previamente los lugares disponibles para cada carrera. Debido a que el número de aspirantes ha sobrepasado los límites de cobertura, principalmente en el área de ciencias sociales y administrativas, se han originado presiones y protestas para que quienes lo demanden logren aceptación en la carrera elegida. Las instituciones han ofrecido para tales estudiantes otras carreras como segunda opción en las que la oferta es mayor que la demanda (ANUIES, 1999).

Las estrategias que propone la Secretaria de Educación Superior (SES) para hacer frente al reto de la cobertura es crear nuevas instituciones y programas de educación superior; ampliar las instituciones y programas existentes; propiciar un uso más eficiente de la capacidad instalada y ampliar la oferta educativa no escolarizada. Para cumplir con estos retos se propone impulsar la apertura de instituciones educativas públicas y privadas que contribuyan al mejoramiento de las demandas económicas y sociales del entorno, atendiendo la demanda del mercado de trabajo, a través de una estrecha vinculación con los requerimientos de los sectores productivo y social, para impulsar el desarrollo local y regional. 
El artículo tercero de la Constitución Política de los Estados Unidos Mexicanos, así como la Ley General de Educación de 1993, reformada en 2002, 2004 y 2006, y la Ley para la Coordinación de la Educación Superior, representan el marco jurídico de la educación superior mexicana que incluye las disposiciones para impartir educación de este nivel a través de instituciones privadas (ANUIES, 1999). La Ley General de Educación (LGE) establece que los centros escolares particulares pueden impartir educación en todos los tipos y modalidades. Para ello, deberán obtener la autorización expresa del Estado en el caso de la educación básica o el reconocimiento de validez oficial de estudios (RVOE), para el caso de los demás tipos educativos (UNESCO-IBE, 2010).

Al tercer trimestre de 2013, los datos de la Encuesta Nacional de Ocupación y Empleo (INEGI, 2013) mostraron que el número total de profesionistas con ocupación, en el país, es de 7.3 millones de personas. Las áreas de conocimiento con más profesionistas con trabajo en México tenían la siguiente distribución: el $27.50 \%$ de la población corresponde al área de "económica- administrativa"; el 21.54\% al campo de "ingenierías" y en tercer lugar se ubican las "ciencias sociales", con un $13.81 \%$.

El área con el mayor número de personas ocupadas hasta el ciclo 2012-2013 fue la económica- administrativa. Sin embargo, el porcentaje de ocupación en quienes estudiaron corresponde a un 38.6\% (Servicio Nacional de Empleo, 2014). Existe un porcentaje bajo en derecho, uno muy bajo en administración y gestión de empresas, como en contabilidad y fiscalización, con respecto a la ocupación que estudiaron. Las áreas que muestran el menor número de individuos ocupados son ciencias físico-matemáticas, humanidades y ciencias biológicas. El empleo en el sector agrícola ha disminuido, en el sector industrial ha crecido moderadamente y en el sector de servicios ha aumentado en forma notable.

De mantenerse el mismo comportamiento de la economía en México, la mayoría de aspirantes continuará demandando carreras del área de servicios, comercio e ingeniería manufacturera. Paradójicamente, pese al aumento paulatino en la matrícula en las carreras relacionadas con la actividad física y deportes, es el área con menor desocupación en el país.

En términos generales, la distribución de la estructura de la población económicamente activa en México es muy similar a la que guardan los países desarrollados, por lo que el proceso de terciarización de la economía ha tenido un impacto en los perfiles de formación de personal técnico y profesional. La forma de organización económica y la evolución de los indicadores macroeconómicos se reflejan no solo en el crecimiento del sector servicios -que constituye el principal destino laboral de los profesionistas-, sino también en una creciente importancia del conocimiento técnico ligado a la información, no solo en este sector, sino en la totalidad de las actividades económicas.

La teoría del capital humano establece que a mayor inversión en educación mayor será el desarrollo en las economías abiertas. Schultz (1972) determinó que cualquier aumento de la capacidad, producida por la inversión humana, se convierte en una parte del agente humano y, por lo tanto, no puede ser objeto de venta, pero afecta de manera directa los sueldos y salarios que perciben las personas. En otras palabras, el aumento resultante de los ingresos es consecuente al rendimiento de la inversión en el capital humano, situación que no coincide con la realidad que se vive en México. 
De acuerdo con Villalobos y Pedroza (2009), la limitación de la teoría del capital humano consiste en determinar a la educación como una mercancía, en la cual se debe invertir para obtener ganancias. Desde una perspectiva crítica, señalan que las tasas de retorno y el análisis costo-beneficio son los indicadores que más se han utilizado para sustentar las políticas y estrategias de los gobiernos en el sentido de argumentar los recortes presupuestarios de los que ha sido objeto la educación, promoviendo la participación del sector privado en este ámbito, para atenuar los problemas que enfrenta el financiamiento del sector. Con estas acciones se reafirma la tesis de que la educación es una mercancía, porque sus resultados se miden a través de las ganancias de la inversión realizada, dejando de lado otros alcances o beneficios que propician el desarrollo económico y social de los países con economías emergentes.

Con base en la teoría del capital humano se esperaría que a mayor cantidad de años de escolaridad, mayor aumento de las probabilidades de obtener un empleo bien remunerado; sin embargo, esta teoría se debilita con los datos estadísticos de desempleo del país, donde aproximadamente el $75 \%$ de la población desempleada, tiene estudios de nivel medio superior o superior. De acuerdo con los resultados de la Encuesta Nacional de Ocupación y Empleo (INEGI, 2012), en diciembre de 2012, se registró que el $24.6 \%$ de personas desocupadas no completó los estudios de secundaria, en tanto que las de mayor nivel educativo representaron al $75.4 \%$ de las desocupadas en el país. Se evidencia de manera contundente que actualmente los años de escolaridad no garantizan el empleo ni un alto ingreso.

Althusser (1969) establece que la fuerza de trabajo disponible debe ser "competente", es decir, apta para ser utilizada en el complejo sistema del proceso de producción. El desarrollo de las fuerzas productivas y el tipo de unidad históricamente constitutivo de esas fuerzas productivas en un momento dado determinan que la fuerza de trabajo debe ser diversamente calificada y, por ende, reproducida como tal. Diversamente, o sea, según las exigencias de la división social técnica del trabajo en sus distintos puestos y empleos.

Lester Thurow (1996) critica la idea de considerar la educación como el mejor instrumento para reducir las desigualdades económicas, donde la formación profesional o técnica influye en el mercado de trabajo. De acuerdo con esta teoría, el mercado existe para equilibrar la demanda y la oferta de trabajo, presentando la competencia salarial como la fuerza impulsora del mercado de trabajo. Thurow realizó un profundo análisis empírico del mercado laboral norteamericano durante el periodo 1950-1970, a partir del cual estableció que el mercado de trabajo se caracteriza más por la competencia de los puestos que por los salarios; es decir, la gente, en lugar de buscar los puestos de trabajo, a partir de los puestos de trabajo busca a los sujetos adecuados para desempeñarlos. Actualmente, el diseño curricular más difundido en el nivel superior se orienta al desarrollo de competencias educativas y profesionales, a partir de estándares de desempeño. Esta metodología es recomendada por organismos internacionales como OCDE, así como por especialistas en política educativa y diseño curricular (Camilloni, 2009).

El panorama actual no es alentador para el estudiantado universitario en México, ya que se requiere de una alta inversión en la habilitación y competencia profesional para enfrentar y aprovechar las mejores ofertas de trabajo, las cuales están a la altura de una persona profesional, pero no hay garantías de integrarse inmediatamente a la población 
económicamente activa del país. Por tal razón, la mayoría de las personas jóvenes se resiste a ganar uno o dos salarios mínimos como remuneración al culminar sus estudios de nivel superior, y resulta absurdo emplearse por tan poco, después de tanto tiempo de asistir a la universidad. Algunas personas graduadas toman la decisión de no trabajar o, al menos, no hacerlo en puestos que no tienen relación con su formación.

La persona joven universitaria es consciente de la falta de oportunidades de trabajo, por ello asisten a la universidad con la intención de obtener un título de licenciatura y trabajar en cualquier campo laboral, considerando los estudios de nivel superior como un requisito para obtener un empleo temporal que le permita cubrir las necesidades más básicas. La mayoría piensa que estudiar en la universidad es un requisito social, y considera el título universitario como una condición de estatus, pero cuando requieran integrarse a la población económicamente activa de México o de cualquier país en Latinoamérica o el mundo, el título obtenido no será suficiente, puesto que se requiere demostrar competencia profesional con un alto nivel de desempeño; de lo contrario, simplemente no se extenderán los contratos de trabajo, debido a que ningún ente empleador está en condiciones de tolerar riegos o pérdidas en su empresa. La población universitaria está consciente de la falta de oportunidades de trabajo, por tal motivo, cada vez son más las personas jóvenes que asisten a la universidad con la intención de obtener un título de licenciatura, para trabajar en cualquier campo laboral.

De acuerdo con lo expuesto, se concluye que los empleos mejor remunerados seguirán siendo para las personas que han logrado finalizar sus estudios de nivel superior, quienes estarán en posibilidades de recuperar el tiempo y dinero invertidos en su formación, transformado sus estudios en capital humano; sin embargo, tal recuperación estará en función de la oferta de empleo y del capital social heredado o dado por sus relaciones con los grupos hegemónicos. Resulta la teoría de Thurow más apropiada para explicar la problemática del desempleo que existe en México, así como la sobrepoblación de profesionales, puesto que no es suficiente la inversión en el capital humano para obtener un empleo, si no se cuenta con la oferta laboral pertinente y suficiente.

Se concluye que el panorama profesional en México representa un escenario limitado para el estudiantado universitario, con base en los siguientes argumentos:

a) Un 75\% de las personas desempleadas en México tienen estudios universitarios.

b) La mayoría de las personas profesionales ocupadas o empleadas realizan actividades que no corresponden a su profesión.

c) La inversión en capital humano no representa para muchos profesionistas un proyecto de recuperación económica a corto plazo.

d) La población de jóvenes en edad de estudiar en la universidad se incrementa cada año; sin embargo, las universidades públicas no tienen la capacidad para facilitar el ingreso a la totalidad de sus aspirantes.

e) La mayoría de jóvenes de universidades requiere trabajar para obtener una remuneración económica que permita pagar una universidad privada; no obstante, los sueldos ofrecidos a las personas sin estudios profesionales resultan muy bajos para seguir estudiando. 
Los retos educativos y las exigencias profesionales se incrementan paulatinamente, las personas jóvenes universitarias requieren desarrollar un perfil de egreso cada vez más competitivo, y la posibilidad de realizar estudios de posgrado resulta una alternativa para invertir en capital humano, mientras la oferta de trabajo sea pertinente. Sin embargo, son pocos los que desean continuar sus estudios en un posgrado de calidad para obtener una beca que resuelva sus problemas económicos a falta de empleo, ya que esta es una manera de hacer frente a los desafíos y retos de nuestro tiempo. Hasta ahora, la calidad educativa es, y seguirá siendo en las próximas décadas, una utopía en el escenario mexicano.

\section{Referencias}

Althusser, L. (1969). Ideología y aparatos ideológicos de Estado. Recuperado de http://www. infoamerica.org/documentos_pdf/althusser1.pdf

ANUIES. (1999). La educación superior en el siglo XXI. Lineas estratégicas de desarrollo. Recuperado de http://publicaciones.anuies.mx/pdfs/revista/Revista113_S5A2ES.pdf

Camilloni, A. (2009). Estándares, evaluación y currículo. Archivos de Ciencias de la Educación. 3(3), 58-68. Universidad de Buenos Aires. Recuperado de http://www.fuentesmemoria. fahce.unlp.edu.ar/art_revistas/pr.4082/pr.4082.pdf

CONAPO. (2014). Proyecciones de la proyección de México 2010-2050. Recuperado de http:// www.conapo.gob.mx/es/CONAPO/Proyecciones

INEGI. (2012). Encuesta Nacional de Ocupación y Empleo 2012. Recuperado de http:// webcache.googleusercontent.com/search?q=cache:zQ46hl_s-CAJ:www.inegi.org.mx/ inegi/contenidos/espanol/prensa/Boletines/Boletin/Comunicados/Indicadores\%2520 estructurales\%2520de\%2520ocupacion\%2520y\%2520empleo/2012/agosto/comunica. $\mathrm{doc}+\& \mathrm{~cd}=3 \& \mathrm{hl}=\mathrm{es}-419 \& \mathrm{ct}=\mathrm{clnk} \& \mathrm{gl}=\mathrm{mx}$

INEGI. (2013). Encuesta Nacional de Ocupación y Empleo 2013. Recuperado de http:// www.inegi.org. mx/inegi/contenidos/espanol/prensa/boletines/boletin/Comunicados/ Especiales/2013/Agosto/comunica12.pdf

PROFLEX. (2010). El profesional flexible en la sociedad del conocimiento. Informe resumen de los resultados del Proyecto PROFLEX en Latinoamérica 2010. Recuperado de http:// www.seguimientoegresados.com/documents/Informe_PROFLEX.pdf

Servicio Nacional de Empleo (2014). Tendencias de empelo profesional. Recuperado de http:// www.empleo.gob.mx/es_mx/empleo/tendencias__de_empleo_de_las_carreras_profesi 
Schultz, T. W. (1972). Inversión en capital humano. En M. Blaug (1972) Economía de la educación. Textos escogidos. Madrid: Siglo XXI.

Thurow, L. (1996). El futuro del capitalismo. Barcelona: Ariel.

Tuirán, R. y Muñoz, C. (2009). Fondo para incremento de matrícula 2009. Evaluación interna. México: Subsecretaría de Educación Superior. Recuperado de http://laisumedu. org/showDoc.php?idDoc=1075\&cates $=$ Desempe\%F1o+de+las + UPMs\&idSubCat $=127 \&$ su bcates $=2 .+$ Cobertura $\% 2 \mathrm{C}+$ oferta $+\mathrm{y}+$ demanda\& $\mathrm{ssc}=9 \& \mathrm{~m}=$ mail $\& \mathrm{p}=$ mail 1

United Nations Educational, Scientific and Cultural Organization \& International Bureal of Education (UNESCO-IBE). (2010/11). Datos mundiales de educación (7 ed). Recuperado de http://www.ibe.unesco.org/fileadmin/user_upload/Publications/WDE/2010/pdfversions/Mexico.pdf

Villalobos, G. y Pedroza, R. (2009). Perspectiva de la teoría del capital humano acerca de la relación entre educación y desarrollo económico. Tiempo de Educar, 10(20), 273306. México: Universidad Autónoma del Estado de México. Recuperado de http://www. redalyc.org/articulo.oa?id=31112987002 